\title{
The Dynamic Nature of Top-Down Signals Originating from Prefrontal Cortex: A Combined fMRI-TMS Study
}

\author{
Taraz G. Lee ${ }^{1}$ and Mark D’Esposito ${ }^{1,2}$ \\ ${ }^{1}$ Department of Psychology and ${ }^{2} \mathrm{Helen}$ Wills Neuroscience Institute, University of California at Berkeley, Berkeley, California, 94110
}

\begin{abstract}
The prefrontal cortex (PFC) is proposed to be the source of top-down signals that can modulate extrastriate visual processing in accordance with behavioral goals, yet little direct causal evidence for this hypothesis exists. Using theta burst transcranial magnetic stimulation, we disrupted PFC function in human participants before performing a working memory task during fMRI scanning. PFC disruption decreased the tuning of extrastriate cortex responses, coinciding with decrements in working memory performance. We also found that activity in the homologous PFC region in the nonstimulated hemisphere predicted performance following disruption. Specifically, those participants with greater homologous PFC activity and greater connectivity between this region and extrastriate cortex were the most resistant to PFC disruption. These findings provide evidence for a compensatory mechanism following insults to the brain, and insight into the dynamic nature of top-down signals originating from the PFC.
\end{abstract}

\section{Introduction}

Working memory refers to the temporary retention of information that was just experienced but no longer exists in the external environment, or was just retrieved from long-term memory (Baddeley, 1986; D'Esposito, 2007). Because of the capacity constraints of working memory (Cowan, 2005), task-relevant information must be effectively encoded for further maintenance by filtering out competing task-irrelevant information (Vogel et al. 2005). Numerous single-unit recording studies in nonhuman primates (Fuster and Alexander, 1971; Miller et al., 1996) and fMRI studies in humans (Curtis and D'Esposito, 2003) have demonstrated that the prefrontal cortex (PFC) and extrastriate cortex exhibit persistent activity during visual working memory delay tasks (Fuster and Jervey, 1982; Miyashita and Chang, 1988; Miller et al., 1993; Postle et al., 2003; Ranganath et al., 2004). Similarly, there is abundant evidence that neural activity is enhanced in those extrastriate cortical regions that encode relevant stimuli (Fuster, 1990; Duncan et al., 1997; Kanwisher and Wojciulik, 2000) and suppressed in regions that represent irrelevant stimuli (Kastner et al., 1998; Gazzaley et al., 2005). Based on this work, it is postulated that the PFC provides top-down signals to extrastriate cortex that selectively enhance the processing and active maintenance of goal-relevant sensory information (Knight et al., 1999; Miller and D'Esposito, 2005).

Although there are numerous studies that suggest the PFC interacts with extrastriate cortex to subserve working memory, the vast majority offer only indirect evidence in support of this

\footnotetext{
Received Jan. 31, 2012; revised Sept. 5, 2012; accepted Sept. 7, 2012.

Author contributions: T.G.L. and M.D. designed research; T.G.L. performed research; T.G.L. analyzed data; T.G.L. and M.D. wrote the paper.

This work was supported by the National Institutes of Health Grants MH63901 and NS40813. We thank J. Lim for his help with running the participants through the experiment and E. M. Nomura for her assistance in data analysis. Correspondence should be addressed to Taraz G. Lee at the above address. E-mail: tarazlee@gmail.com.

DOI:10.1523/JNEUROSCI.0627-12.2012

Copyright $\odot 2012$ the authors $\quad 0270-6474 / 12 / 3215458-09 \$ 15.00 / 0$
}

claim. Studies examining correlated neural activity between disparate brain regions cannot provide causal evidence that the PFC influences posterior brain regions. However, several studies with experimentally induced lesions in monkeys have provided direct causal evidence for functional interactions between the PFC and extrastriate cortex (Fuster et al., 1985; Tomita et al., 1999; Monosov et al., 2011). In humans, event-related potential (ERP) recordings in patients with PFC lesions have shown that an intact PFC is needed for effective sensory processing in posterior visual areas during working memory tasks (Chao and Knight, 1998; Barceló et al., 2000).

Previously, attempts have been made to better characterize the nature of top-down signals that influence extrastriate cortex by using transcranial magnetic stimulation (TMS) to perturb frontal cortex function in healthy humans while simultaneously or subsequently recording activity in extrastriate cortex using both EEG (Taylor et al., 2007; Morishima et al., 2008; Zanto et al., 2011) and fMRI (Ruff et al., 2006; Miller et al., 2011; Higo et al., 2011). While these studies demonstrated that disruption of PFC activity can modulate the magnitude of activity in posterior visual areas, only Miller et al. (2011) demonstrated that PFC disruption also modulates the selectivity of neural representations coding visual categories. However, in that study, the relationship between behavioral performance and modulation of neural selectivity could not be demonstrated. In monkeys, studies that stimulated or inactivated cortical activity using either electrical current or pharmacological manipulations demonstrated that the frontal eye fields (FEFs) alter neural tuning in the extrastriate region V4 (Moore and Armstrong, 2003; Monosov et al., 2011). However, top-down effects on higher-level representations, such as stimulus categories, have yet to be demonstrated. Thus, the aim of the current study was to determine the causal role of a more rostral area of PFC (e.g., critical for the working memory) in biasing category representations stored in extrastriate cortex. We accomplished this by administering TMS in healthy humans to disrupt PFC function before fMRI scanning during performance of a 

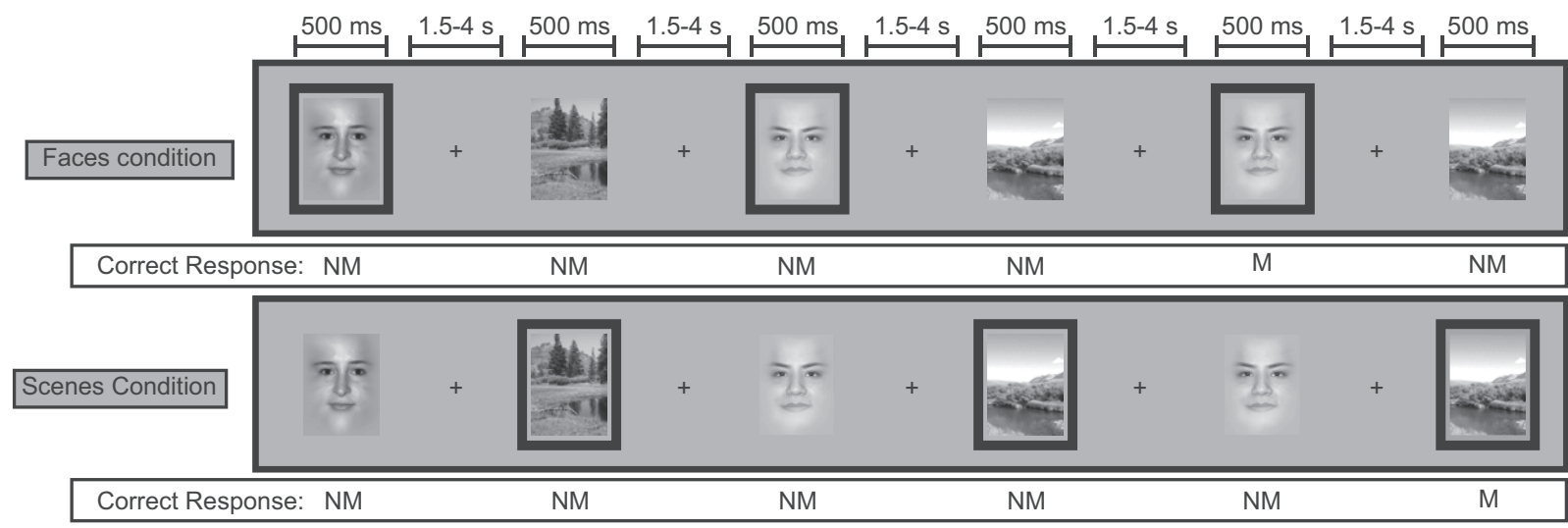

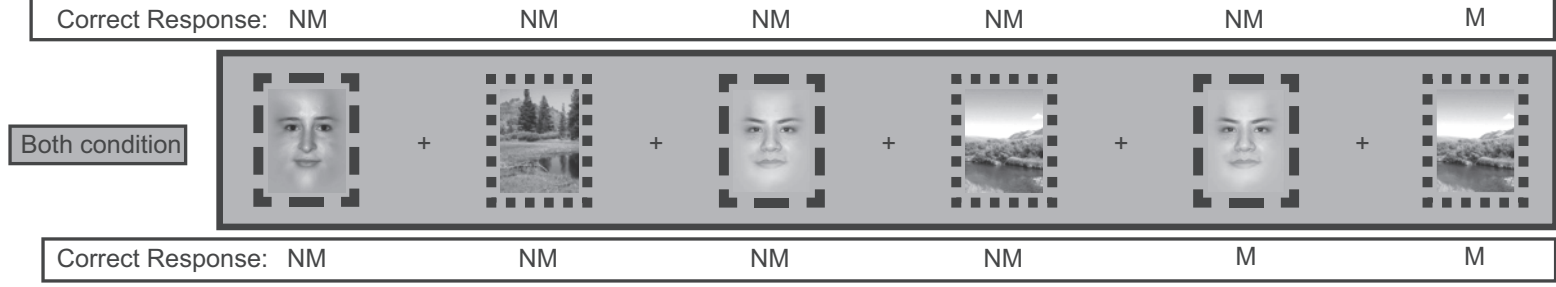
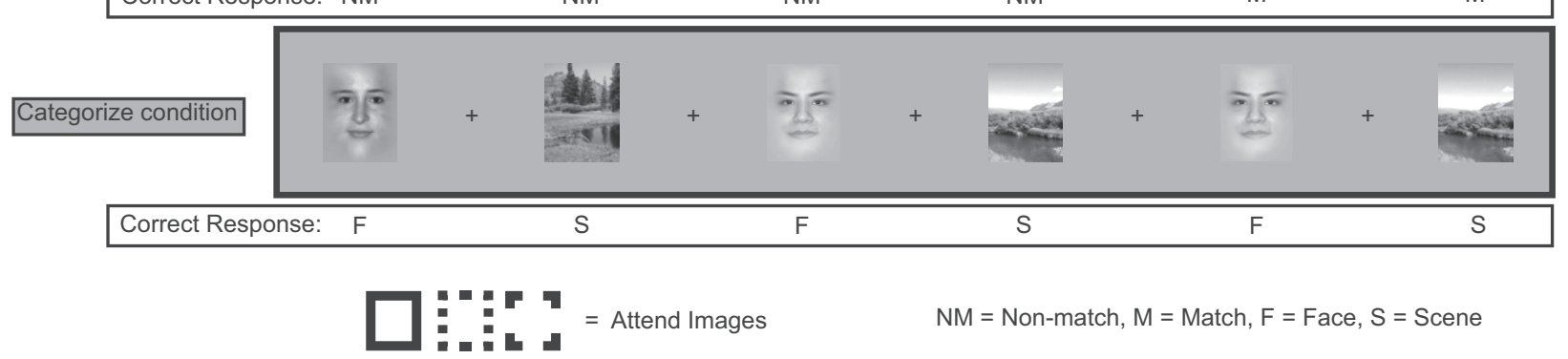

$\mathrm{NM}=$ Non-match, $\mathrm{M}=$ Match, $\mathrm{F}=$ Face, $\mathrm{S}=$ Scene

Figure 1. Structure of the behavioral task. Participants were prompted at the beginning of a block which stimulus categories to remember (FACES, SCENES, BOTH) or identify (CATEGORIZE). In the FACES, SCENES, BOTH conditions, on each trial, participants manually responded whether or not the image currently on the screen was a repeat of the last image of the relevant stimulus category. Each image had to be maintained across all intertrial intervals (1.5-4.5s) and intervening distracter images (up to 3). In this illustration of the task, a box surrounding a stimulus indicates that it is from the category that must be remembered; these boxes were not presented to the participant. In the CATEGORIZE condition, participants simply responded whether the current image was a face or a scene.

working memory task. If PFC is the source of top-down modulation, disruption of this region should alter the gain and the tuning of task-relevant representations within extrastriate cortex and lead to impaired performance on the task.

\section{Materials and Methods}

Participants. Twelve right-handed subjects (five males, seven females; age range, 18-29) participated in the experiment. All participants gave their written informed consent before the study and received monetary compensation for their participation.

Experimental timeline. Each participant underwent three separate fMRI acquisition sessions while performing a cognitive task. The first session was used to obtain a T1-weighted anatomical MRI scan and to acquire data to define regions of interest (ROIs) for use in subsequent analyses. This first session was used to train participants on the task. Before the second and third sessions (counterbalanced across participants), TMS was applied either over a left inferior frontal gyrus (LIFG) region that was functionally defined from each participant's initial scan, or a control brain region (somatosensory cortex). After each TMS session, participants were taken directly to the MR room and into the scanner. On average there was a 9 min delay from the end of TMS and the acquisition of the first functional image.

Task design. While being scanned during each of the three sessions, participants performed five runs of a modified N-back task each consisting of four blocks of 20 trials for each behavioral condition (Fig. 1). In this task, images of faces and scenes were sequentially presented, and participants were instructed to selectively attend to and maintain images from the relevant category while ignoring those from the irrelevant category, or to attend and maintain both categories. At the beginning of each condition block, participants were given an instructional prompt to inform them which category of stimuli was to be remembered and which were to be ignored: (1) ATTEND FACES (attend to faces and ignore scenes), (2) ATTEND SCENES (attend to scenes and ignore faces), (3) ATTEND BOTH (respond to both faces and scenes), (4) CATEGORIZE (indicate whether the current image is a face or a scene, with no attempt to remember them). On each trial, participants were presented with an image of either a face or a natural scene and had to indicate by button press whether or not the current image matched the image last seen that was of the same category. If an image was of the unattended category, participants had to simply press the "nonmatch" button. An identical number of faces and scenes were presented in each block and the number of "distractor" images from the unattended category between successive images of the attended category ranged between 0 and 3. Images were presented on the screen at fixation for $500 \mathrm{~ms}$ with a varying intertrial interval of $1.5-4.5 \mathrm{~s}$.

Transcranial magnetic stimulation. For all experiments, participants were seated in a comfortable chair. Electromyography was recorded using electrodes from the right first dorsal interosseous (FDI) muscle of the dominant hand in all participants. TMS was applied using a handheld figure-eight coil with an outer winding diameter of $70 \mathrm{~mm}$ (Magstim). All pulses were delivered using a Magstim rapid stimulator connected to four booster modules that produce biphasic pulses. Stimulation was delivered over primary motor cortex approximately over an area representing the hand with the coil placed tangentially to the scalp with the handle pointing posteriorly. The motor hand area was defined as the location on the scalp where magnetic stimulation produced the largest motorevoked potential (MEP) from the contralateral FDI when the participant was relaxed. The stimulation intensity was defined in relation to the 
active motor threshold (AMT) of the participant. The AMT was defined as the minimum intensity of single-pulse stimulation required to produce an MEP on $>5$ of 10 trials from the contralateral FDI while the participant was maintaining a voluntary contraction of $\sim 20 \%$ of maximum in the FDI. Raw EMG signal on the screen was provided as visual feedback to the participant to help maintain a constant muscle contraction of the correct force.

The stimulation targets were localized using Brainsight, a computerized frameless stereotaxy system (Rogue Research). This system uses an infrared camera to monitor the positions of reflective markers attached to the participant's head. Head locations are related in real time to the participant's previously acquired structural MRI data after the data are coregistered to a set of anatomical locations. Reflective markers are attached to the coil and the participant, so that relative positions of the coil to the head (and the MRI) can be tracked, allowing precise positioning of the coil with respect to previously chosen MRI locations.

Many repetitive TMS studies have used $1 \mathrm{~Hz}$ stimulation to cause transient reductions in cortical excitability that outlast the period of stimulation. However, the reported effects at this stimulation rate are short lasting (usually 15 min or less) and often weak and highly variable (Gangitano et al., 2002). In contrast, continuous theta burst TMS is a more recent approach developed to address this shortcoming (Huang et al., 2005). Continuous theta burst TMS parameters used in the current study were identical to those described by Huang et al. (2005). Theta burst TMS consists of $50 \mathrm{~Hz}$ trains of three TMS pulses repeated every $200 \mathrm{~ms}$ continuously over a period of $40 \mathrm{~s}(600$ pulses total) and has been shown to depress activity in the stimulated region for up to $60 \mathrm{~min}$ following stimulation. This longer duration of altered excitability is ideally suited for studying the effects of TMS with fMRI given that many imaging studies require repeated acquisition runs lasting $>30 \mathrm{~min}$.

Stimulation sites. The LIFG was chosen for TMS because it was determined to be a likely source of top-down modulation of early visual areas based on functional connectivity analyses we performed on data collected during performance of the cognitive task used in the current study (our unpublished data). While many studies have opted to use the vertex of the skull as a control region for TMS, it often falls between cerebral hemispheres and is only likely to mimic the sensation of stimulation and not actually stimulate brain tissue. Thus, to control for changes due to nonspecific stimulation of brain tissue as well as the scalp sensation of stimulation, the control region used in this study was the left postcentral gyrus (PCG). This site was not activated by our task in any participant.

Functional MRI acquisition and preprocessing. MR data were acquired with a Siemens 3 Tesla scanner with a 12-channel head coil. Functional data were obtained using a one-shot $\mathrm{T}_{2}{ }^{*}$-weighted echoplanar imaging sequence sensitive to blood oxygenation level-dependent (BOLD) contrast (TR, $1000 \mathrm{~ms}$; TE, $32 \mathrm{~ms}$; field of view, $230 \mathrm{~mm}$; matrix size, $64 \times 64$; in-plane resolution, $3.5 \times 3.5 \mathrm{~mm}$ ). Each functional volume contained 18 contiguous 3 -mm-thick axial slices separated by a $0.5 \mathrm{~mm}$ interslice gap. Whole-brain MP Flash $\mathrm{T}_{1}$-weighted scans were acquired for anatomical localization. Functional data were realigned to the first volume acquired and spatially smoothed with a $4 \mathrm{~mm}$ full-width at halfmaximum Gaussian kernel.

Behavioral analysis. Mean response times (RTs) and accuracy rates were computed for each participant for all behavioral conditions (ATTEND FACES, ATTEND SCENES, ATTEND BOTH, and CATEGORIZE). The effect of TMS on the task was evaluated by a repeated- measures ANOVA on the RTs and accuracy, with the stimulation site (control and LIFG) and the behavioral condition as within-subject factors. Two-tailed $t$ tests were used to compare results between the two stimulation sites where appropriate.

Univariate fMRI analysis. Task-dependent changes in the BOLD signal were modeled with independent regressors for each behavioral condition (ATTEND FACES, ATTEND SCENES, ATTEND BOTH, and CATEGORIZE) and each stimulus type (face or scene). These regressors were made by convolving a gamma function with a vector containing the onset times for each trial type. Trials with incorrect behavioral responses were modeled separately and excluded so that the resulting analyses considered only trials in which participants made a correct response. Statistical contrasts assessing the main effect of attention (gain effect) for each condition were computed as weighted sums of the estimated $\beta$ coefficients (ATTEND FACES, attended face stimuli vs ignored face stimuli; ATTEND SCENES, attended scene stimuli vs ignored scene stimuli; ATTEND BOTH, all attended face and scene stimuli vs all CATEGORIZE stimuli). Each run was mean centered and detrended for linear and polynomial trends using linear least squares. Maps of the parameter estimates ( $\beta$ values) were computed from the GLM from each session and normalized on a subject-by-subject basis to each individual's anatomical scan. Mean parameter estimates for each regressor were also calculated within each functionally defined ROI for each subject. Two-tailed, paired sample $t$ tests were used to test for the significance of differences across groups and across task contrasts for each ROI.

ROIs. LIFG, fusiform face area (FFA), and parahippocampal place area (PPA) ROIs were functionally defined using different contrasts from data from each participant's first fMRI session. LIFG ROIs were defined on the basis of an attentional gain effect. We selected the most statistically significant cluster of activity in the LIFG using a contrast of all attended images versus all ignored images, regardless of stimulus type across all behavioral conditions (Fig. 2). Bilateral FFA ROIs were created by selecting the cluster of activity in the fusiform gyrus of each hemisphere that 

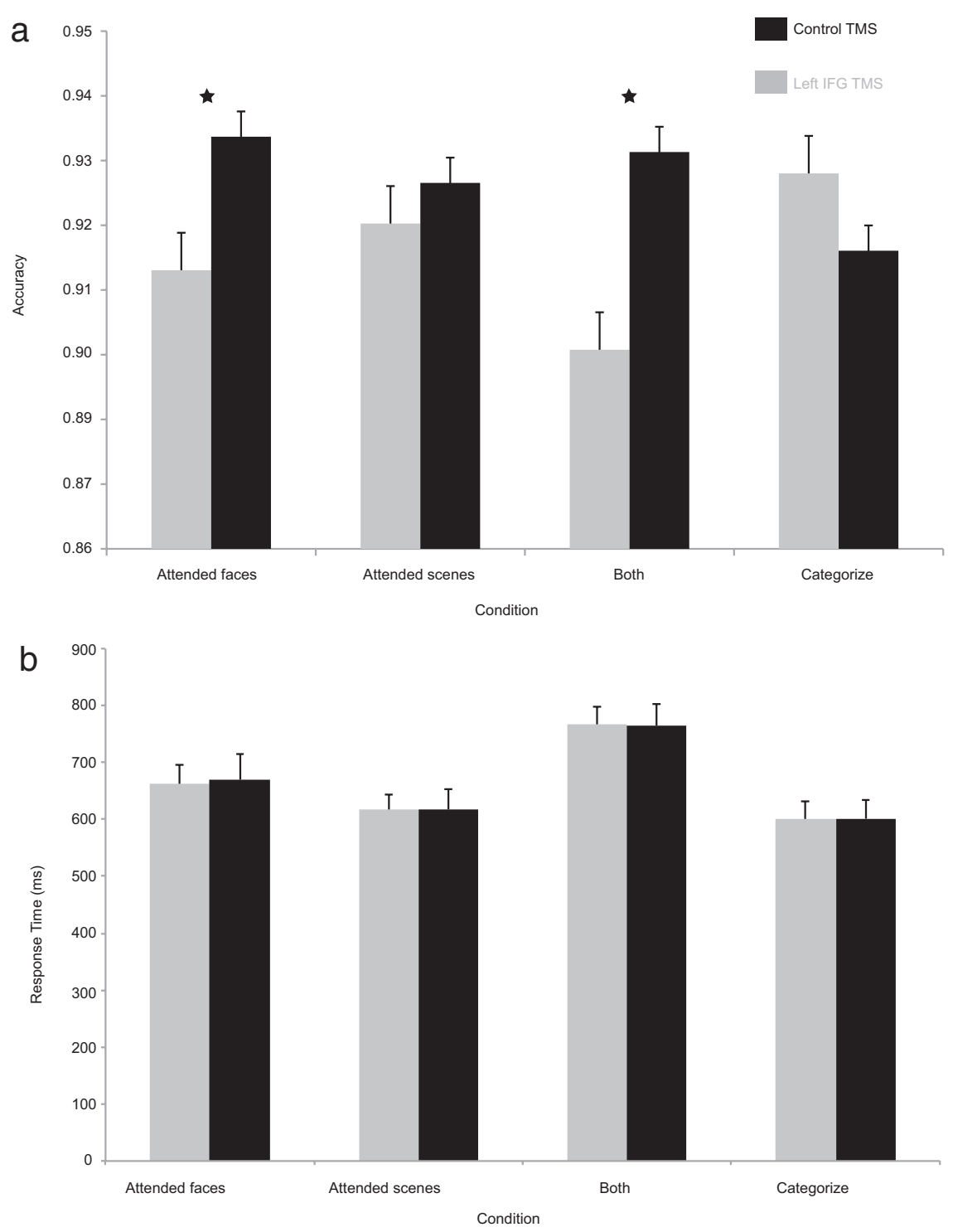

Figure 3. Behavioral results. $\boldsymbol{a}$, Accuracy during the FACES and BOTH conditions of the task was reduced after TMS to LIFG compared to TMS of a control region. $\boldsymbol{b}$, TMS to LIFG did not affect response times in any condition. Data are represented as $\pm S E M$. ${ }^{*} p<0.05$.

exhibited the strongest preference to face versus scene stimuli across all behavioral conditions, as assessed by a $t$ test. Similarly, bilateral PPA ROIs were created by selecting the cluster of voxels in the parahippocam$\mathrm{pal} /$ lingual gyrus that exhibited the strongest preference to scene versus face stimuli across all behavioral conditions. Right inferior frontal gyrus (RIFG) ROIs were created by taking the mirror flip of each participants functionally defined LIFG ROI. Left PCG ROIs were anatomically defined with reference to the Duvernoy (1999) brain atlas. Each participant's left PCG was identified on the anatomical scan, and ROIs were drawn as spheres with a radius of $5 \mathrm{~mm}$ centered $10 \mathrm{~mm}$ away from the midline and $5 \mathrm{~mm}$ from the top edge of the brain.

Functional connectivity analysis. Functional connectivity maps were created on a subject-by-subject basis using a $\beta$ series connectivity analysis described previously by Rissman et al. (2004). This approach allows us to determine correlations on a trial-by-trial basis between brain regions as opposed to blocks of trials. This analysis produces a whole-brain map of Pearson's $r$ values for each subject that were subsequently transformed using Fisher's $r$-to- $z$ transformation.

Category selectivity analysis (spatial correlations). Previous work has shown that while extrastriate cortex seems to have distinct modules that appear to process certain categories of visual stimuli preferentially (e.g., faces and scenes), there is evidence that the representation of this information is actually quite widely distributed and overlapping (Haxby et al. 2001; Op de Beeck et al. 2008). To determine the distinctiveness of scene and face representations in extrastriate cortex, we used a spatial correlation analysis. In this analysis, the similarity of patterns of neural activity evoked by the task after TMS to the two different stimulation sites was assessed. Calculating the correlation between spatial patterns of activity evoked by the task under the different TMS conditions offers another method for evaluating the change in neural activity as a result of frontal stimulation since spatial correlations are relatively insensitive to differences in the magnitude of evoked activity that might occur between the two stimulation conditions.

In this analysis, large functional ROIs comprised of the union of all scene-selective and face-selective voxels across the entire temporal and occipital lobe during each participant's initial scan were defined as each participant's left and right extrastriate cortex. These ROIs were subdivided into a set of new ROIs comprising the most task-active voxels (top 30, 40, $50,60,70,80,90,100,110$, and 120 voxels) as defined by a contrast of attended images versus ignored images. For each stimulation condition (IFG or control), the $\beta$ value for the corresponding covariate was obtained for each voxel within each ROI. These values were translated into a linear vector for each separate ROI for each condition (Aguirre, 2007). Correlations (Kendall's $\tau$ ) of the evoked activity between the two stimulus categories (face and scene) within each task were calculated. These correlations are the spatial correlations of the pattern of activity evoked by faces versus scenes. For each participant, the difference in the correlation value between IFG stimulation and control stimulation was calculated and used as an index of the change in category selectivity due to frontal stimulation.

The significance of the observed difference in correlations was assessed by creating a simulated distribution of correlation values via bootstrapping by randomizing the values within each subset of voxels before calculating each correlation. Shuffling was done 10,000 times per subject for each stimulation condition. A difference score was calculated for each bootstrapping iteration, and this resulted in a Gaussian distribution of difference values. Difference values more extreme than $95 \%$ of the distribution were taken to be significant.

Brain-behavior correlation analysis. Brain-behavior correlation plots were created by plotting the mean $t$ value of a contrast of interest or $z$ value (from the connectivity analysis) from a given ROI for each participant as a function of his mean RT or accuracy for the given contrast of interest. Correlations between brain activity and behavior were explored with Spearman's coefficient $r$.

\section{Results}

\section{Behavioral analyses}

A repeated-measures ANOVA on accuracy and RTs with TMS site (IFG, control), condition (ATTEND FACES, ATTEND SCENES, ATTEND BOTH, CATEGORIZE), and stimulus type (faces, scenes) as within-subject factors was performed. This revealed a main effect of condition (accuracy, $F_{(3,33)}=3.88, p<$ 

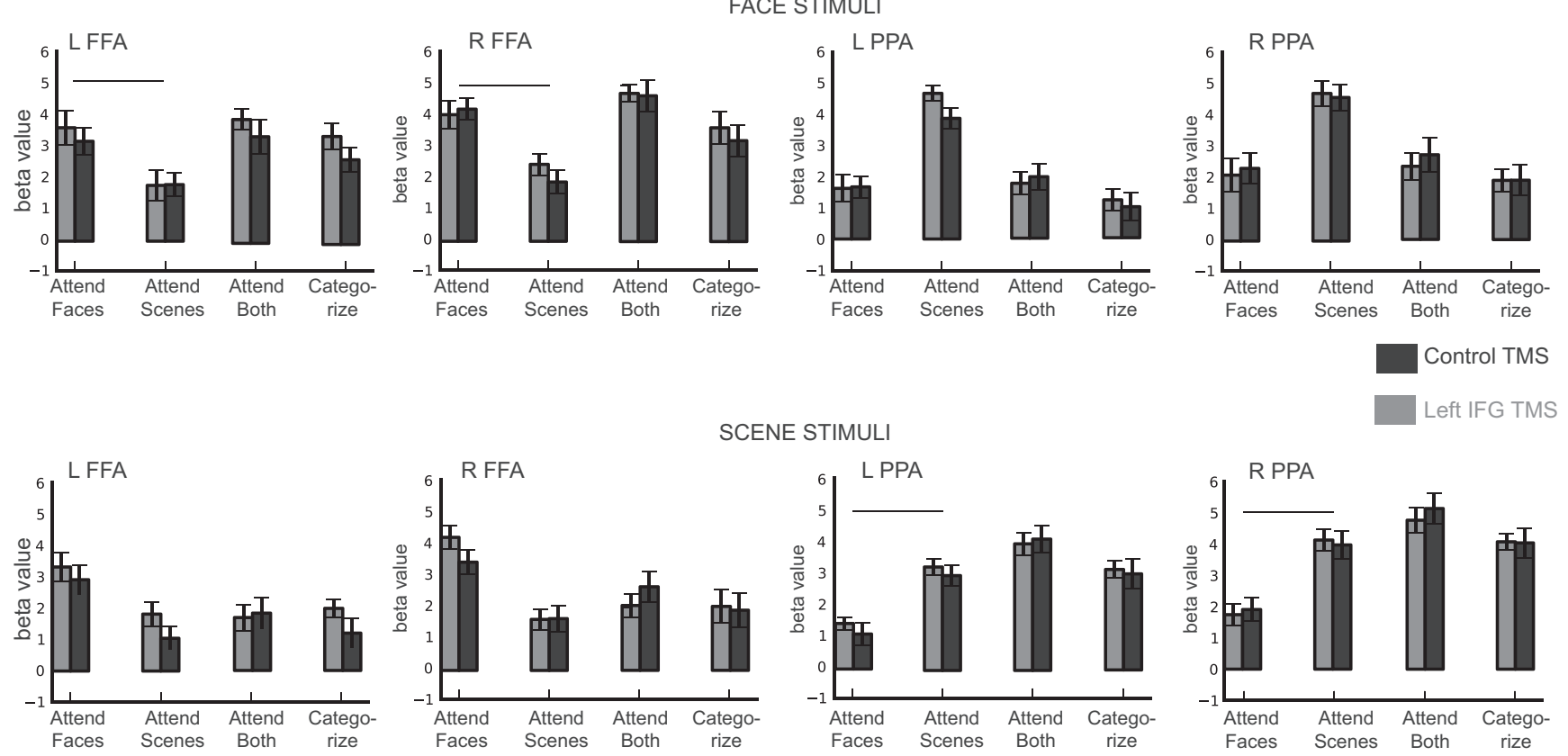

Figure 4. Univariate results from the FFA and PPA ROIs during each of the task conditions for each stimulus type. While the gain effect was present in both FFA (left, $t_{(11)}=5.42, p<0.0005 ;$ right, $t_{(11)}=6.47, p<0.0001$ ) and PPA (left, $t_{(11)}=5.12, p<0.0005$; right, $\left.t_{(11)}=7.17, p<0.0001\right)$, there were no significant TMS effects. Bars denote the gain effect. Error bars indicate SEM.

0.05 ; RT, $\left.F_{(3,33)}=42.23, p<0.001\right)$. Participants had the slowest RT and worst accuracy during the BOTH condition, followed by the FACES and SCENES conditions, then the CATEGORIZE condition (Fig. 3; linear contrasts, accuracy, $F_{(1,11)}=10.764, p<0.01 ; \mathrm{RT}, F_{(1,11)}=$ $48.94, p<0.001)$. The TMS site by condition interaction was also significant (accuracy, $\left.F_{(3,33)}=2.92, p<0.05\right)$. As predicted, performance suffered after LIFG stimulation when compared to control stimulation, but not in all conditions equally (Fig. 3; linear contrast, accuracy, $\left.F_{(1,11)}=6.647, p<0.03\right)$. Accuracy was significantly worse after LIFG TMS only during the BOTH $\left(t_{(11)}=2.41, p<0.05\right)$ and FACES conditions $\left(t_{(11)}=2.35, p<\right.$ $0.05)$. Finally, accuracy in responding "nonmatch" to irrelevant stimuli (faces in the SCENES condition and scenes in the FACES condition) was similar across the TMS conditions (LIFG TMS, 98.3\%; control TMS, 98.7\%; $\left.t_{(11)}=0.68, p=0.52\right)$ suggesting that participants were able to keep track of the task rule following PFC stimulation.

\section{Univariate fMRI analyses}

Consistent with our previous work (Gazzaley et al., 2005), all participants showed significantly enhanced FFA activity when face images were attended (i.e., relevant) versus when they were ignored (i.e., irrelevant), and significantly enhanced PPA activity when scene images were attended versus ignored (Fig. 4). The magnitude of this gain effect during trials in which stimuli were relevant versus irrelevant can be considered an index of topdown modulation. Thus, we examined the effect of LIFG TMS on this metric, defined as the $t$ value calculated from the statistical contrast of the BOLD response to attended versus unattended stimuli within the FFA (for face stimuli) and PPA (for scene
Control TMS

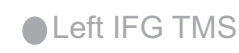

\section{Left IFG}

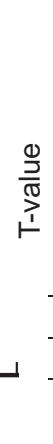

Right IFG

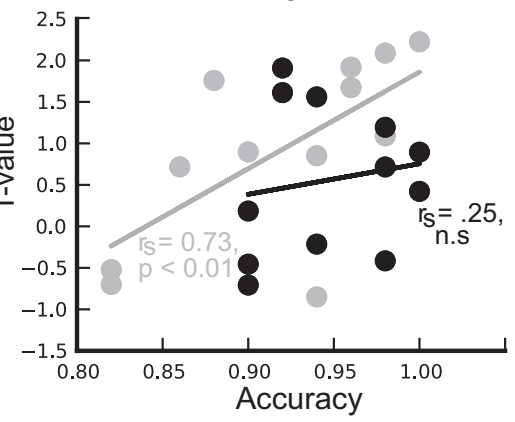

Figure 5. Correlation plots of IFG activity versus task accuracy during the FACES condition. Following TMS to the control region, LIFG activity, but not RIFG activity, correlated with accuracy. Following TMS to LIFG, the opposite pattern was observed—LIFG activity no longer correlated with accuracy, whereas RIFG activity and accuracy became correlated.

stimuli). Subsequently, we will refer to this metric as the "gain effect." In the two behavioral conditions in which LIFG TMS, compared to control TMS, caused worse performance (ATTEND FACES condition and ATTEND BOTH condition), the gain effect within the FFA was reduced after LIFG TMS. The gain effect in PPA during the ATTEND SCENES condition was not reduced following LIFG TMS. None of these effects, however, were significant at the group level, likely due to significant variability across subjects. This individual variability in the BOLD data prompted us to determine whether it could be accounted for by individual differences in the effect of TMS on behavioral performance.

\section{Brain-behavior correlation results}

To further explore the relationship between TMS effects on brain activity and behavioral performance, the gain effect in each frontal and extrastriate ROI (i.e., left and right IFG, left and right FFA) during the conditions in which behavioral 
performance was affected by TMS (i.e., ATTEND FACES and ATTEND BOTH conditions) was correlated with participants' behavior.

Following TMS to the control region, the LIFG was the only ROI that exhibited a significant relationship between the gain effect during the FACES condition ( $\beta$ value for attended faces vs ignored faces) and behavior (RT and accuracy). Specifically, there was a significant positive correlation between task performance and the magnitude of the gain effect in LIFG in response to attended face stimuli; that is, the most accurate participants exhibited the greatest gain effect in LIFG $\left(r_{\mathrm{s}}=0.79, p<0.01\right.$; Fig. 5). Consistent with our prediction that the LIFG is the source of top-down signals modulating the gain of extrastriate cortical activity, this brain-behavior relationship during the FACES condition was not significant after LIFG TMS $\left(r_{\mathrm{s}}=0.27, p=0.40\right)$. There were no significant correlations between behavior and the gain effect in any region during the BOTH or SCENES condition.

To explore possible compensatory mechanisms supported by the nonstimulated hemisphere, we also examined the relationship between behavior and the gain effect in the RIFG. While there was no relationship between the RIFG gain effect and performance in the FACES condition after control stimulation $\left(r_{\mathrm{s}}=0.25, p=0.43\right)$, the RIFG gain effect was positively correlated with accuracy following LIFG stimulation $\left(r_{\mathrm{s}}=0.73, p<0.01\right.$; Fig. 5$)$. To further investigate the contribution of RIFG function to successful performance following LIFG TMS, we assessed the relationship between functional connectivity between RIFG and right FFA and the TMSinduced change in accuracy during the FACES condition. A change in accuracy was calculated as the difference between performance after control TMS and that after PFC TMS (LIFG, control TMS). Functional connectivity between the RIFG and the right FFA after control stimulation successfully predicted this change in accuracy following LIFG TMS $\left(r_{s}=\right.$ $0.545, p<0.07$; Fig. $6 a$ ). Furthermore, a median split of the participants based on the change in performance between LIFG TMS and control TMS showed that poorer performers had significantly less connectivity between RIFG and right FFA after control TMS $\left(t_{(6)}=2.594, p<0.05\right.$; Fig. $\left.6 b\right)$. These findings suggest that preserved behavioral performance following LIFG disruption depends on the ability of the nonstimulated right hemisphere to compensate for altered LIFG function.

\section{Spatial correlation results}

The results presented above suggest that PFC can modulate the gain of extrastriate cortical activity; however, we also sought evidence that PFC can modulate the tuning of extrastriate cortex representations. If true, LIFG TMS should lead to decreased category selectivity, which would manifest as higher spatial correlations between stimulus-evoked activity within extrastriate cortex in response to attended images of one category (face or scene) and ignored images of the other category.

As predicted, LIFG TMS, compared to control TMS, significantly increased the spatial correlation between the patterns of extrastriate cortex activity evoked by attended faces and ignored scenes in the FACES condition $(p<0.05$ via permutation testing). These increases were observed in both left and right extrastriate cortex regardless of how many voxels were included in the analysis (within a range of 30-120 voxels; Fig. 7a). In contrast, our measure of within-category spatial correlations (patterns of activity evoked by attended faces vs activity evoked by ignored
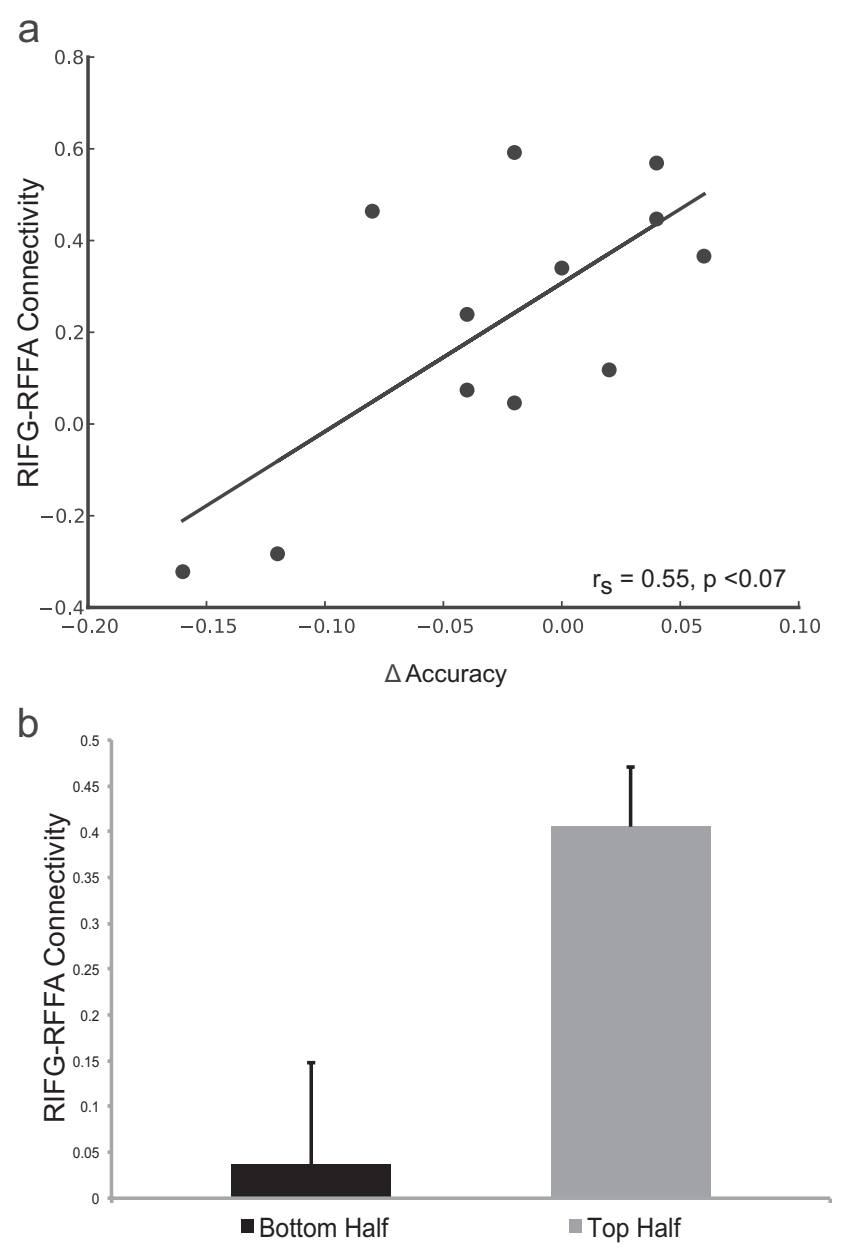

Figure 6. A higher level of connectivity between the RIFG and the RFFA is predictive of less of a decrement in accuracy on the task following TMS to LIFG. $\boldsymbol{a}$, Correlation plot of RIFG-RFFA connectivity and TMS-related change in accuracy during the FACES condition. $\boldsymbol{b}$, Participants whose accuracy suffered the most as a result of LIFG TMS (bottom half of participants based on a median split of behavior) had significantly lower levels of RIFG-RFFA connectivity after control TMS. Error bars represent SEM. $\Delta$ equals IFG TMS minus control TMS.

faces) was not affected by LIFG stimulation ( $p>0.1$ via permutation testing; Fig. $7 b$ ).

To assess how this change in selectivity to face and scene stimuli following LIFG TMS affected working memory performance, we plotted the change in accuracy in the FACES condition for each participant who exhibited a decrement in performance following LIFG TMS against their change in spatial correlation. Indices of the TMS-induced change in accuracy and spatial correlation were calculated as the difference between LIFG and control TMS. As predicted, the greater the increase in spatial correlation between extrastriate cortical activity evoked by faces and scenes as a result of LIFG TMS, the greater the decrement in accuracy participants exhibited. Again, when averaged across hemispheres this relationship was significant across a range of ROI sizes (70-120 voxels, $r_{\mathrm{s}}=-0.72-0.89, p<0.05$; Fig. 8). Moreover, it was also significant when the stimulated hemisphere was examined alone (within a range of $30-70$ voxels, $r_{\mathrm{s}}=-0.71-$ $0.86, p<0.05$ ) and approached significance in the nonstimulated hemisphere.

\section{Discussion}

To assess the causal influence of the PFC on top-down modulation of posterior visual processing regions, we used continuous 

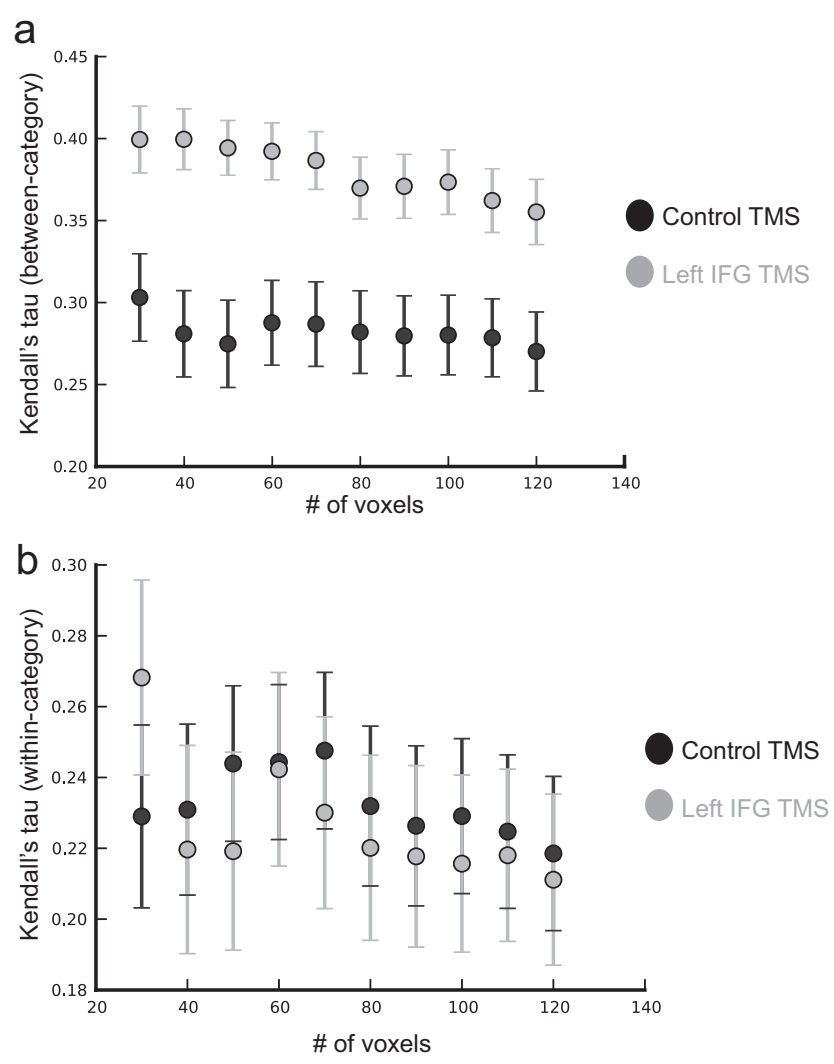

Figure 7. Category selectivity (as measured by Kendall's $\tau$ ) in bilateral extrastriate cortex. $\boldsymbol{a}$, Spatial correlation between attended faces and ignored scenes during the FACES condition of the task. TMS to the LIFG led to increases in spatial correlation between evoked activity to the face and scene stimuli, which indicates a decrease in category selectivity in the underlying cortex. $\boldsymbol{b}$, The spatial correlation between attended faces and ignored faces (within-category correlation) was not affected by LIFG TMS. Error bars represent SEM.

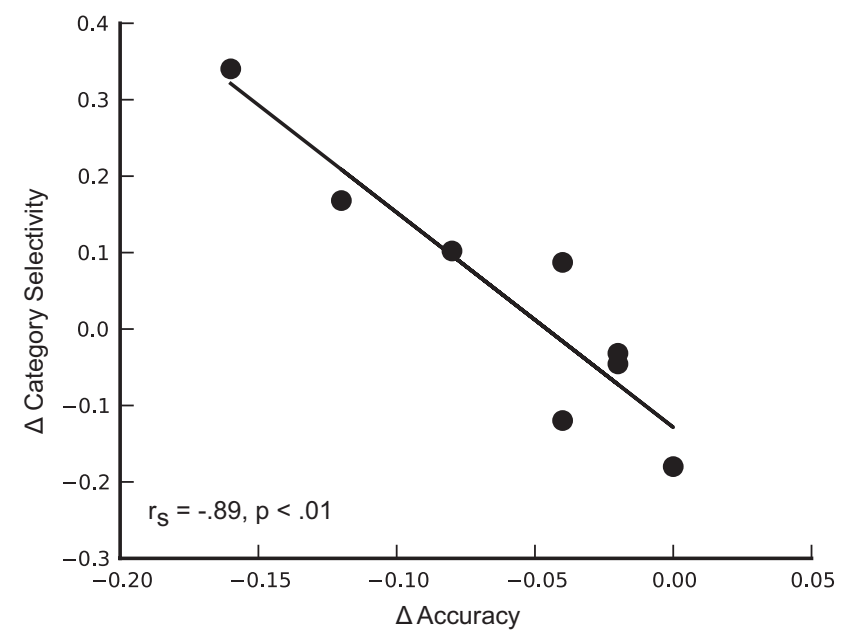

Figure 8. Correlation plot between TMS-induced change in category selectivity (as measured by Kendall's $\tau$ ) in bilateral extrastriate cortex (120 voxel ROl size) and change in accuracy during in the FACES condition. Following TMS to LIFG, an increase in spatial correlations (indicative of a decrement in category selectivity) is predictive of a greater decrement in accuracy on the task. $\Delta$ equals IFG TMS minus control TMS.

theta burst TMS to disrupt function in the LIFG during a working memory task. Our results show that after stimulation, LIFG function was altered such that activity in this region was no longer predictive of performance on a working memory task. We also show that this frontal disruption leads to a change in the selectivity of extrastriate cortical activity, and that this change corresponds with a decrement in behavioral performance. Our data also suggest that those participants who are best able to maintain a high level of performance after LIFG stimulation recruit the homologous RIFG as a possible compensation for disrupted function in the stimulated hemisphere. Additionally, the participants who had greater connectivity between the RIFG and the right FFA before PFC TMS were the most resistant to disruption, that is, they exhibited the least decrement in behavioral performance.

While studies using functional connectivity analyses to examine network interactions during top-down modulation are informative (Miller and D'Esposito, 2005), this method is correlational and does not provide strong evidence for causal interactions. Previously, two separate human studies have shown that the FEFs play a causal role in attentional processing by combining TMS with EEG (Taylor et al., 2007; Morishima et al., 2008). Both studies found that perturbing function in the FEFs leads to decreased task-evoked activity at posterior electrode sites. In monkeys, Moore and Armstrong (2003) observed that electrical stimulation of FEFs produces enhancement of the visual response of V4 neurons to target stimuli. After infusion of a dopaminergic agonist into FEFs, both the magnitude and orientation selectivity of V4 responses was reduced. Ekstrom et al. (2009) found that electrical stimulation of FEFs in monkeys during simultaneous fMRI scanning produced an amplification of extrastriate cortical activity at low-stimulus contrast but sharpened responses at high-stimulus contrast levels. Finally, Monosov et al. (2011) observed, after deactivation of FEF function in monkeys with muscimol, reduced object-selective responses in inferotemporal cortex and impaired performance on a visual search task.

A previous study in humans using combined TMS and EEG during performance of a working memory task found that top-down signals similar to those found in FEFs originate from a more rostral frontal region, the inferior frontal junction (IFJ). Gain changes in activity in electrodes recording activity in early visual areas were found after IFJ disruption (Zanto et al., 2011). In our previous work (Miller et al., 2011) and the present study, by combining TMS with fMRI, we replicated and extended these findings by demonstrating that top-down signals originating from PFC (e.g., anterior to the FEFs) act via both gain and tuning mechanisms. Moreover, the better spatial resolution of fMRI allowed us to determine that the PFC in a homologous region in the nonstimulated hemisphere can provide compensatory top-down signals following PFC disruption in the opposite hemisphere. Previously, another study used TMS to disrupt PFC function before the performance of a selective attention task performed during fMRI (Higo et al., 2011). PFC TMS diminished top-down modulation of extrastriate cortical activity, but did not impact the bottom-up activation of these same regions. However, these TMS-induced activity changes were not associated with changes in behavioral performance. In contrast, our results demonstrate that the magnitude of gain and selectivity changes in extrastriate cortex following PFC disruption is predictive of subsequent behavioral performance on a working memory task.

In addition to providing evidence that top-down signals from PFC can modulate the gain of activity in early visual areas, we also sought to determine whether top down signals could modulate neural tuning. To investigate tuning, we measured the selectivity of BOLD responses in early visual areas to different categories of visual stimuli (i.e., faces vs scenes). Previous work in nonhuman 
primates has shown that neurons in inferotemporal cortex respond preferentially to specific colors during a delayed match-tosample task, and that disrupting PFC function can lead to decrements in behavior by abolishing the color-dependent differences in firing rate among neurons in inferotemporal cortex such that these neurons no longer exhibited their previous color preference and responded to different colored stimuli similarly (Fuster et al., 1985). In another fMRI study (Miller et al., 2011), we demonstrated that disrupting PFC function can reduce the selectivity of responses to face and scene stimuli in extrastriate cortex, but this change in tuning could not be linked to behavioral performance. The current study replicates this finding, but we also demonstrate for the first time in humans that altered neural selectivity disrupts behavioral performance on a working memory task.

Studies of patients with brain lesions have shown that working memory function is left relatively unimpaired following unilateral PFC damage (D'Esposito and Postle, 1999). Previous work suggested that increased activity in the intact hemisphere in these patients may serve as a compensatory response for the loss of function in damaged tissue (Corbetta et al., 2005; Nudo, 2007; Voytek et al., 2010). Consistent with these findings, we found that only after TMS to the left IFG, activity within the homologous right IFG predicted successful performance on the working memory task. A previous study of patients with diffuse axonal injury due to brain trauma reported a similar finding (Turner et al., 2011). In that study, control participants activated a left-lateralized PFC network that correlated with working memory function, whereas homologous right PFC activity did not. However, in patients with diffuse axonal injury, right PFC activity did correlate with working memory function. The researchers attributed this pattern of findings to "unmasking of extant, but behaviorally latent" brain networks supporting working memory function rather functional reorganization per se (i.e., recruitment of brain networks not engaged by controls on the task). We believe that a similar mechanism also explains our findings.

Other patient studies have suggested that there are transcallosal interactions between homologous regions in the two hemispheres, which are likely inhibitory, and that unilateral damage (or TMS disruption) can cause increased excitation of the undamaged hemisphere (Sprague, 1966; Kapur, 1996; Shimizu et al., 2002; Kobayashi et al., 2004). Thus, studies of patients with focal lesions that demonstrated that TMS disruption of the contralesional hemisphere ameliorates behavioral deficits may act by releasing the perilesional tissue from transcallosal inhibition (Oliveri et al., 1999; Mansur et al., 2005; Naeser et al., 2011). In our study, we propose that TMS disruption of left IFG function may have led to increased activity in the homologous right IFG, leading to a release of a dormant (latent) network that could support working memory function. However, the time scale of the effects we observed is significantly different from that of the effects observed in patients with chronic lesions either from stroke or traumatic brain injury. Previous studies using electrophysiological recordings in nonhuman primates demonstrated that PFC neurons are highly adaptable and can code for newly learned stimulus categories (Freedman et al., 2001), learn new stimulus-response associations over a matter of trials (Asaad et al., 1998), and even represent two different types of information over different delay periods of a single trial of a working memory task (Rao et al., 1997). The compensatory effects we observed immediately after left IFG disruption are consis- tent with these prior findings and provide further support that PFC function can adapt on a relatively short time scale in response to behavioral demands.

\section{References}

Aguirre GK (2007) Continuous carry-over designs for fMRI. Neuroimage 35:1480-1494. CrossRef Medline

Asaad WF, Rainer G, Miller EK (1998) Neural activity in the primate prefrontal cortex during associative learning. Neuron 21:1399-1407. CrossRef Medline

Baddeley A (1986) Working memory. Oxford, UK: Oxford UP.

Barceló F, Suwazono S, Knight RT (2000) Prefrontal modulation of visual processing in humans. Nat Neurosci 3:399-403. CrossRef Medline

Chao LL, Knight RT (1998) Contribution of human prefrontal cortex to delay performance. J Cogn Neurosci 10:167-177. CrossRef Medline

Corbetta M, Kincade MJ, Lewis C, Snyder AZ, Sapir A (2005) Neural basis and recovery of spatial attention deficits in spatial neglect. Nat Neurosci 8:1603-1610. CrossRef Medline

Cowan N (2005) Working memory capacity. Hove, UK: Psychology.

Curtis CE, D'Esposito M (2003) Persistent activity in the prefrontal cortex during working memory. Trends Cogn Sci 7:415-423. CrossRef Medline

D'Esposito M (2007) From cognitive to neural models of working memory. Philos Trans R Soc 362:761-772. CrossRef

D'Esposito M, Postle BR (1999) The dependence of span and delayedresponse performance on prefrontal cortex. Neuropsychologia 37:89101. CrossRef

Duncan J, Humphreys G, Ward R (1997) Competitive brain activity in visual attention. Curr Opin Neurobiol 7:255-261. CrossRef Medline

Duvernoy, H.M. (1999) The human brain: surface, three-dimensional sectional anatomy with MRI, and blood supply, Ed 2. New York: Springer Wien.

Freedman DJ, Riesenhuber M, Poggio T, Miller EK (2001) Categorical Representation of visual stimuli in the primate prefrontal cortex. Science 291:312-316. CrossRef Medline

Fuster JM (1990) Infer temporal units in selective visual attention and short-term memory. J Neurophysiol 64:681-697. Medline

Fuster JM, Alexander GE (1971) Neuron activity related to short-term Memory. Science 173:652-654. CrossRef Medline

Fuster JM, Jervey JP (1982) Neuronal firing in the inferotemporal cortex of the monkey in a visual memory task. J Neurosci 2:361-375. Medline

Fuster JM, Bauer RH, Jervey JP (1985) Functional interactions between inferotemporal and prefrontal cortex in a cognitive task. Brain Res 330:299_ 307. CrossRef Medline

Gangitano M, Valero-Cabré A, Tormos JM, Mottaghy FM, Romero JR, Pascual-Leone A (2002) Modulation of input-output curves by low and high frequency repetitive transcranial magnetic stimulation of the motor cortex. Clin Neurophysiol 113:1249-1257. CrossRef

Gazzaley A, Cooney JW, McEvoy K, Knight RT, D’Esposito M (2005) Topdown enhancement and suppression of the magnitude and speed of neural activity. J Cogn Neurosci 17:507-517. CrossRef Medline

Higo T, Mars RB, Boorman ED, Buch ER, Rushworth MF (2011) Distributed and causal influence of frontal operculum in task control. Proc Natl Acad Sci U S A 108:4230-4235. CrossRef Medline

Haxby JV, Ida Gobbini M, Furey ML, Ishai A, Schouten JL, Pietrini P (2001) Distributed and overlapping representations of faces and objects in ventral temporal cortex. Science 293:2425-2430. CrossRef Medline

Huang YZ, Edwards MJ, Rounis E, Bhatia KP, Rothwell JC (2005) Theta burst stimulation of the human motor cortex. Neuron 45:201-206. CrossRef Medline

Kanwisher N, Wojciulik E (2000) Visual attention: insights from brain imaging. Nat Rev Neurosci 1:91-100. Medline CrossRef

Kapur N (1996) Paradoxical functional facilitation in brain-behavior research: A critical review. Brain 119:1775-1790. CrossRef Medline

Kastner S, De Weerd P, Desimone R, Ungerleider LG (1998) Mechanisms of directed attention in the human extrastriate cortex as revealed by functional MRI. Science 282:108-111. CrossRef Medline

Knight RT, Staines WR, Swick D, Chao LL (1999) Prefrontal cortex regulates inhibition and excitation in distributed neural networks. Acta Psychol 101:159-178. CrossRef Medline

Kobayashi M, Hutchinson S, Théoret H, Schlaug G, Pascual-Leone A (2004) Repetitive TMS of the motor cortex improves ipsilateral sequential simple finger movements. Neurology 62:91-98. CrossRef Medline 
Mansur CG, Fregni F, Boggio PS, Riberto M, Gallucci-Neto J, Santos CM, Wagner T, Rigonatti SP, Marcolin MA, Pascual-Leone A (2005) A sham stimulation-controlled trial of rTMS of the unaffected hemisphere in stroke patients. Neurology 64:1802-1804. CrossRef Medline

Miller BT, D’Esposito M (2005) Searching for "the top" in top-down control. Neuron 48:535-538. CrossRef Medline

Miller BT, Vytlacil J, Fegen D, Pradhan S, D’Esposito M (2011) The prefrontal cortex modulates category selectivity in human extrastriate cortex. J Cogn Neurosci 23:1-10. CrossRef Medline

Miller EK, Li L, Desimone R (1993) Activity of neurons in the anterior inferior temporal cortex during a short-term memory task. J Neurosci 13: 1460-1478. CrossRef Medline

Miller EK, Erickson CA, Desimone R (1996) Neural mechanisms of visual working memory in prefrontal cortex of the macaque. J Neurosci 16: 5154-5167. Medline

Miyashita Y, Chang HS (1988) Neuronal Correlate of pictorial short-term memory in the primate temporal cortex. Nature 331:68-70. CrossRef Medline

Monosov IE, Sheinberg DL, Thompson KG (2011) The effects of prefrontal cortex inactivation on object responses of single neurons in the inferotemporal cortex during visual search. J Neurosci 31:15956-15961. CrossRef Medline

Moore T, Armstrong KM (2003) Selective gating of visual signals by microstimulation of frontal cortex. Nature 421:370-373. CrossRef Medline

Morishima Y, Akaishi R, Yamada Y, Okuda J, Toma K, Sakai K (2008) Taskspecific signal transmission from prefrontal cortex in visual selective attention. Nat Neurosci 12:85-91. CrossRef Medline

Naeser MA, Martin PI, Theoret H, Kobayashi M, Fregni F, Nicholas M, Tormos JM, Steven MS, Baker EH, Pascual-Leone A (2011) TMS suppression of right pars triangularis, but not pars opercularis, improves naming in aphasia. Brain and Language 119:206-213. CrossRef Medline

Nudo RJ (2007) Postinfarct cortical plasticity and behavioral recovery. Stroke 38:840-845. CrossRef Medline

Oliveri M, Rossini PM, Traversa R, Cicinelli P, Filippi MM, Pasqualetti P, Tomaiuolo F, Caltagirone C (1999) Left frontal transcranial magnetic stimulation reduces contralesional extinction in patients with unilateral right brain damage. Brain 122:1731-1739. CrossRef Medline

Op de Beeck HP, Haushofer J, Kanwisher NG (2008) Interpreting fMRI data: maps, modules and dimensions. Nat Rev Neurosci 9:123-135. CrossRef Medline
Postle BR, Druzgal TJ, D’Esposito M (2003) Seeking the neural substrates of visual working memory storage. Cortex 39:927-946. CrossRef Medline

Ranganath C, Cohen MX, Dam C, D'Esposito M (2004) Inferior temoral, prefrontal, and hippocampal contributions to visual working memory maintenance and associative memory retrieval. J Neurosci 24:3917-3925. CrossRef Medline

Rao SC, Rainer G, Miller EK (1997) Integration of what and where in the primate prefrontal cortex. Science 276:821-824. CrossRef Medline

Rissman J, Gazzaley A, D’Esposito M (2004) Measuring functional connectivity during distinct stages of a cognitive task. Neuroimage 23: 752-763. CrossRef Medline

Ruff CC, Blankenburg F, Bjoertomt O, Bestmann S, Freeman E, Haynes JD, Rees G, Josephs O, Deichmann R, Driver J (2006) Concurrent TMS-fMRI and psychophysics reveal frontal influences on human retinotopic visual cortex. Curr Biol 16:1479-1488. CrossRef Medline

Shimizu T, Hosaki A, Hino T, Sato M, Komori T, Hirai S, Rossini PM (2002) Motor cortical disinhibition in the unaffected hemisphere after unilateral cortical stroke. Brain 125:1896-1907. CrossRef Medline

Sprague JM (1966) Interaction of cortex and superior colliculus in mediation of visually guided behavior in the cat. Science 153:1544-1547. CrossRef Medline

Taylor PC, Nobre AC, Rushworth MF (2007) FEF TMS affects visual cortical activity. Cereb Cortex 17:391-399. Medline

Tomita H, Ohbayashi M, Nakahara K, Hasegawa I, Miyashita Y (1999) Topdown signal from prefrontal cortex in executive control of memory retrieval. Nature 401:699-703. CrossRef Medline

Turner GR, McIntosh AR, Levine B (2011) Prefrontal compensatory engagement in TBI is due to altered functional engagement of existing networks and not functional reorganization. Front Sys Neurosci 5:1-12. CrossRef Medline

Vogel EK, McCollough AW, Machizawa MG (2005) Neural measures reveal individual differences in controlling access to working memory. Nature 438:500-503. CrossRef Medline

Voytek B, Davis M, Yago E, Barceló F, Vogel EK, Knight RT (2010) Dynamic neuroplasticity after human prefrontal cortex damage. Neuron 68: 401-408. CrossRef Medline

Zanto T, Rubens M, Thangavel A, Gazzaley A (2011) Causal role of the prefrontal cortex in top-down modulation of visual processing and working memory. Nat Neurosci 14:656-661. CrossRef Medline 